\title{
Optimization Models for the Radio Planning of Wireless Mesh Networks
}

\author{
Edoardo Amaldi, Antonio Capone, Matteo Cesana, and Federico Malucelli \\ Politecnico di Milano, Dipartimento Elettronica ed Informazione, Milano, Italy \\ $\{$ amaldi, capone, cesana, malucell\}@elet.polimi.it
}

\begin{abstract}
In this paper we propose novel optimization models for the planning of Wireless Mesh Networks whose objective is to minimize the network installation cost, while providing full coverage to wireless mesh clients. Our mixed integer linear programming models aim at selecting the number and positions of mesh routers and access points, while taking into account in an accurate way traffic routing, interference, rate adaptation, and channel assignment. We provide the optimal solutions of the proposed problem formulations on a set of realistic-size instances and discuss the effect of different parameters on the characteristics of the planned networks.
\end{abstract}

\section{Introduction}

The network devices composing Wireless Mesh Networks (WMNs) 1 are of three types: Mesh Routers (MRs), Mesh Access Points (MAPs) and Mesh Clients (MCs). The functionality of both the MRs and the MAPs is twofold: they act as classical access points towards the MCs, whereas they have the capability to set up a Wireless Distribution System (WDS) by connecting to other mesh routers or access points through point to point wireless links. Both MRs and MAPs are often fixed and electrically powered devices. Furthermore, the MAPs are geared with some kind of broadband wired connectivity (LAN, ADSL, fiber, etc.) and act as gateways toward the wired backbone. MCs are users terminals connected to the network through MAPs or MRs.

Several parameters concur to the determination of a general wireless mesh network effectiveness including the number of radio interfaces for each device, the number of available radio channels, the access mechanism, the routing strategies and the specific wireless technology used to implement the mesh paradigm. All these parameters are de facto degrees of freedom the network designer can exploit to deploy an effective WMN, thus optimization criteria are needed for the tuning of such parameters.

To this end, many works have appeared in the literature with the purpose of providing optimized protocols for WMNs. So et al. propose in [2] a multichannel $\mathrm{MAC}$ protocol in the case single interface transceivers are used, whereas reference [3] analyzes those networks where even multiple radio interface per wireless node can be used adapting the channel access protocol. Das et al. propose two 
Integer-Linear programming models to solve the fixed channel assignment problem with multiple radio interfaces [4], whilst [5] and [6] address the problems of channel assignment and routing jointly, providing different formulations to the optimization problem.

The most of the previously published work assumes a given network topology, i.e., the general approach tends to optimize the channel assignment and/or the routing assuming given positions for the MRs and the MAPs. On the other hand, the purpose of the present work is to model the radio planning problem of WMNs, providing quantitative methods to optimize number, positions and coordination of MRs and MAPs and the overall topology of the network.

The problem of planning WMNs differs from that of planning other wireless access networks, such as cellular systems [7] or WLANs 8. In the latter cases, network planning involves selecting the locations in which to install the base stations or access points, setting their configuration parameters (emission power, antenna height, tilt, azimuth, etc.), and assigning channels so as to cover the service area and to guarantee enough capacity to each cell [9].

In the case of WMNs, each candidate site can host either MAPs or MRs, which have different installation costs. Roughly speaking, MAPs are more expensive than MRs since they must be directly connected to the wired backbone and might be more powerful than MRs in terms of both processing and transmission capabilities. Moreover, the traffic to/from the wired backbone has to be routed on a path connecting the MR to one MAP at least. In this context, capacity limits of radio links among MRs and between MRs and MAPs play a key role since the traffic routed on a link must not exceed its capacity.

The resulting network design problem must simultaneously consider the radio coverage of users, like in classical radio planning for wireless access networks [9], and the traffic routing, like in the design of wired networks [10].

Very few previous works consider the problem of planning WMNs or, more in general, fixed multi-hop wireless network. The main attempt to address the problem appears in [1] where the focus is on locating internet transit access points (MAPs in the terminology adopted in this paper) in wireless neighborhood network. Heuristic solutions and a lower bound are provided. Since the positions of all the other nodes (MRs) is given and such nodes are also the only traffic ending points in the network, the problem considered in [11] is actually a subproblem of that proposed in this paper since the coverage part is not included. Moreover, the interference model is based on the number of hops crossed by flows, while the model considered in this paper is a fluidic version [12] of the protocol interference model [14. The problem in 11] was originally proposed in 13 . where, on the other hand, a time division multiple access is assumed and the slot scheduling optimization is included in the model.

In this work we propose an optimization model for the problem of planning WMNs based on mathematical programming which takes into account both the local and the multihop connectivity requirements. The problem is NP-hard but it can be solved to the optimum for realistic size instances. We provide 
optimal solutions for a set of synthetic instances and discuss the effect of different parameters on the characteristics of the solution.

Our work is organized as follows: Section 2 gives the formulation of the proposed model and comments on its main features, whilst Section 3 reports numerical results. Concluding remarks are given in Section 4

\section{Wireless MESH Network Planning}

For the sake of simplicity, we define a basic version of the model for the WMN planning problem neglecting interference, adaptive modulation and multiple channels, and then we extend it step-by-step. Let us consider the network description presented in Figure 1

Similarly to the coverage problems commonly considered for wireless access networks [9], let $S=\{1, \ldots, m\}$ denote the set of CSs and $I=\{1, \ldots, n\}$ the set of TPs. A special node $N$ represents the wired backbone network. The cost associated to installing a MR in $\mathrm{CS} j$ is denoted by $c_{j}$, while the additional cost required to install a MAP in $\mathrm{CS} j$ is denoted by $p_{j}, j \in S$. The total cost for installing a MAP in CS $j$ is therefore given by $\left(c_{j}+p_{j}\right)$. Traffic generated by TP $i$ is given by parameter $d_{i}, i \in I$. The traffic capacity of the wireless link between CSs $j$ and $l$ is denoted by $u_{j l}, j, l \in S$, while the capacity of the radio access interface of CS $j$ is denoted by $v_{j}, j \in S$. The sequence $S_{i}$ of CSs that can cover TP $i$ is calculated for all TPs considering a non increasing order of received signal strength, $S_{i}=\left\{j_{1}^{(i)}, j_{2}^{(i)}, \ldots, j_{L_{i}}^{(i)}\right\}, i \in I$.

According to TPs and CSs location and propagation information the connectivity parameters can be calculated. Let $a_{i j}, i \in I, j \in S$, be the coverage parameters:

$$
a_{i j}=\left\{\begin{array}{l}
1 \text { if a MAP or MR in } \mathrm{CS} j \text { cover TP } i \\
0 \text { otherwise, }
\end{array}\right.
$$

and $b_{j l}, j, l \in S$, the wireless connectivity parameters:

$$
b_{j l}=\left\{\begin{array}{l}
1 \text { if } \operatorname{CS} j \text { and } l \text { can be connected with a link } \\
0 \text { otherwise. }
\end{array}\right.
$$

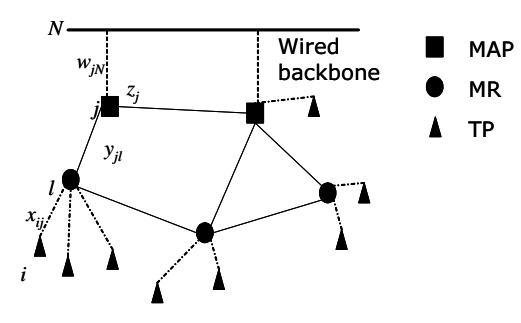

Fig. 1. WMN planning problem description 
Decision variables of the problem include TP assignment variables $x_{i j}, i \in$ $I, j \in S:$

$$
x_{i j}=\left\{\begin{array}{l}
1 \text { if } \mathrm{TP} i \text { is assigned to } \mathrm{CS} j \\
0 \text { otherwise, }
\end{array}\right.
$$

installation variables $z_{j}, j \in S$ :

$$
z_{j}=\left\{\begin{array}{l}
1 \text { if a MAP or a MR is installed in CS } j \\
0 \text { otherwise, }
\end{array}\right.
$$

wired backbone connection variables $w_{j N}, j \in S$ (if $z_{j}=1, w_{j N}$ denote if $j$ is connected to the wired network $N$, i.e. if it is a MAP or a MR):

$$
w_{j N}=\left\{\begin{array}{l}
1 \text { if a MAP is installed in } \mathrm{CS} j \\
0 \text { otherwise }
\end{array}\right.
$$

wireless connection variables $y_{j l}, j, l \in S$ :

$$
y_{j l}=\left\{\begin{array}{l}
1 \text { if there is a wireless link between } \operatorname{CS~} j \text { and } l \\
0 \text { otherwise, }
\end{array}\right.
$$

and finally flow variables $f_{j l}$ which denote the traffic flow routed on link $(j, l)$, where the special variable $f_{j N}$ denotes the traffic flow on the wired link between MAP $j$ and the backbone network.

Given the above parameters and variables, the WMN planning problem can be stated as follows:

$$
\min \sum_{j \in S}\left(c_{j} z_{j}+p_{j} w_{j N}\right)
$$

s.t.

$$
\begin{aligned}
& \sum_{j \in S} x_{i j}=1 \quad \forall i \in I \\
& x_{i j} \leq z_{j} a_{i j} \quad \forall i \in I, \forall j \in S \\
& \sum_{i \in I} d_{i} x_{i j}+\sum_{l \in S}\left(f_{l j}-f_{j l}\right)-f_{j N}=0 \quad \forall j \in S \\
& f_{l j}+f_{j l} \leq u_{j l} y_{j l} \quad \forall j, l \in S \\
& \sum_{i \in I} d_{i} x_{i j} \leq v_{j} \quad \forall j \in S \\
& f_{j N} \leq M w_{j N} \quad \forall j \in S \\
& y_{j l} \leq z_{j}, y_{j l} \leq z_{l} \quad \forall j, l \in S \\
& y_{j l} \leq b_{j l} \quad \forall j, l \in S \\
& z_{j_{\ell}^{(i)}}+\sum_{h=\ell+1}^{L_{i}} x_{i j_{h}^{(i)}} \leq 1 \quad \forall \ell=1, \ldots, L_{i}-1, \forall i \in I \\
& x_{i j}, z_{j}, y_{j l}, w_{j N} \in\{0,1\} \quad \forall i \in I, \forall j, l \in S
\end{aligned}
$$


The objective function (11) accounts for the total cost of the networks including installation costs $c_{j}$ and costs related to the connection of MAP to the wired backbone $p_{j}$. If for some practical reason only a MR and not a MAP can be installed in CS $\mathrm{j}$, the corresponding variable $w_{j N}$ is set to zero. Constraints (2) provide full coverage of all TPs, while constraints (3) are coherence constraints assuring respectively that a TP $i$ can be assigned to CS $j$ only if a device (MAP or MR) is installed in $j$ and if $i$ is within the coverage set of $j$.

Constraints (4) define the flow balance in node $j$. These constraints are the same as those adopted for classical multicommodity flow problems. The term $\sum_{i \in I} d_{i} x_{i j}$ is the total traffic related to assigned TPs, $\sum_{l \in S} f_{l j}$ is the total traffic received by $j$ from neighboring nodes, $\sum_{l \in S} f_{j l}$ is the total traffic transmitted by $j$ to neighboring nodes, and $f_{j N}$ is the traffic transmitted to the wired backbone. Even if these constraints assume that traffic from TPs is transmitted to the devices to which they are assigned and that this traffic is finally delivered by the network to the wired backbone, without loss of generality we can assume that $d_{i}$ accounts for the sum of traffic in the uplink (from TPs to the WMN) and in the downlink (from WMN to the TPs) since radio resources are shared in the two directions.

Constraints (5) impose that the total flow on the link between device $j$ and $l$ does not exceed the capacity of the link itself $\left(u_{j l}\right)$. Constraints (6) impose for all the MCs' traffic serviced by a network device (MAP or MR) not to exceed the capacity of the wireless link used for the access, whilst constraints (7) forces the flow between device $j$ and the wired backbone to zero if device $j$ is not a MAP. The parameter $M$ is used to limit the capacity of the installed MAP.

Constraints (8) and (9) defines the existence of a wireless link between CS $j$ and CS $l$, depending on the installation of nodes in $j$ and $l$ and wireless connectivity parameters $b_{j l}$. The constraints expressed by (10) force the assignment of a TP to the best CS in which a MAP or MR is installed according to a proper sorting criteria (such as the received signal strength), whilst constraints (11) defines the decision variables of the model to assume binary values only. Obviously, the above model is NP-hard since it includes the set covering and the multi-commodity flow problems as special cases.

The model defined above considers fixed transmission rates for both the wireless access interface and for the wireless distribution system, and it will be referred to as Fixed Rate Model (FRM) throughout the paper. The FRM can be easily extended to endorse transmission rate adaptation. As to the wireless distribution system, the rate adaptation can be accounted directly in the variables $u_{j l}$. Rate adaptation in the wireless access network can be accounted in the model with a slight modifications of constraints (6). We consider several concentric regions centered in each CS, assigning to each region a maximum rate value. All the TPs falling in one of these regions can communicate with the node in the CS using the specific rate of the region.

Formally, we can define the set of regions for a given $\mathrm{CS} \mathrm{j} R_{j}^{k}=1, \ldots, K$ and the set $I_{j}^{k} \subset I$ containing all the TPs falling in region $\mathrm{k}$ of CS $\mathrm{j}$. Such sets can be determined for each CS $\mathrm{j}$ using the incidence variable $a_{i j}^{k}$ which is equal to 
1 if TP $i$ falls within region $k$ of the CS $j$ and to zero otherwise. Each of these regions of a given $\mathrm{CS} \mathrm{j}$ is assigned a maximum capacity defined by variable $v_{j}^{k}$. Using such definitions, the FRM can be extended to the case of rate adaptation in the wireless access part of the network by substituting the constraints (6) with the following new constraints:

$$
\sum_{k \in R_{j}} \frac{\sum_{i \in I_{j}^{k}} d_{i} x_{i j}}{v_{j}^{k}} \leq 1 \quad \forall j \in S
$$

The new defined model with constraints (12) will be referred to as Rate Adaptation Model (RAM) throughout the paper.

Both the FRM and the RAM do not consider the effect of the interference on the access capacity and on the capacity of wireless links connecting mesh nodes. However, in practical cases we must take into account interference effect. We focus here on the case of IEEE 802.11 and assume that all MAPs and MRs share the same radio channel for the access part and use another shared channel for the backbone links. Since now the access capacity is shared by all mesh nodes, we can take into account interference quite easily modifying constraints (12) and considering not only the TPs assigned to the node in CS $j$ but all TPs in the coverage range:

$$
z_{j} \sum_{k \in R_{j}} \frac{\sum_{i \in I_{j}^{k}} d_{i}}{v_{j}^{k}} \leq 1 \quad \forall j \in S
$$

The interference limiting effect on the wireless link capacities is more difficult to account for, since it depends on the network topology and the multiple access protocol. Considering the protocol interference model proposed in [14, we can define sets of links that cannot be active simultaneously. These sets depend on the specific multiple access protocol considered. In the case of CSMA/CA, adopted by IEEE 802.11 , each set $C_{j l}$ considers a link $(j, l)$ and includes all links that are one and two hops away in the mesh-network graph (links connecting $j$ and $l$ to their neighbors and their neighbors to the neighbors of their neighbors). To each set we can associate a constraint on the flows crossing its links:

$$
y_{j l} \sum_{(k, h) \in C_{j l}} \frac{f_{k h}}{u_{k h}} \leq 1 \quad \forall j, l \in S
$$

Obviously, by describing the capacity limitation due to set $C_{j l}$ with the constraint on flows crossing its links, we make an approximation since we pass from a discrete model to a fluidic one 12 . The effect of this approximation and the one due to traffic dynamics can be accounted by properly reducing capacity values $u_{k h}$. We estimated through simulation of IEEE 802.11 multi-hop networks that a reduction of $5 \%$ is sufficient to achieve consistent results.

Replacing constraints (5) with (14) and (6) with (13) we get a new model referred to in the paper as Interference Aware Model (IAM). Note that the nonlinear constraints (14) can be easily linearized. 
As a matter of fact, FRMT/RAM and IAM can be considered extreme cases with respect to interference, corresponding respectively to those scenarios where enough channels and radio interfaces are available in the mesh nodes so that interference can be neglected, and to those scenarios where only one channel is available for the mesh backbone. In all the other intermediate scenarios, channel assignment to mesh nodes must be included in the optimization model.

The extension of the model to the multiple channels case is quite straightforward and, due to length constraints, here we just outline the modifications needed. Let us assume that a set $F$ of $Q$ channels is available and that each mesh node is equipped with $B$ radio cards. New installation variables $z_{j}^{q}(q \in F, j \in S)$ must be considered which are equal to one if a mesh node is installed in $j$ and is assigned channel $q$, and to zero otherwise. Also link variables $y_{j l}^{q}$ are extended to include the channel used by link $(j, l)$, as well as flow variables $f_{j l}^{q}$. In the objective function we replace variable $z_{j}$ with new variable $t_{j}$ which is equal to 1 if a mesh node is installed in $j$. Constraints are easily modified to include the new dimension related to channels. In particular, constraints (3)-(5) are replaced with the following:

$$
\begin{gathered}
x_{i j} \leq \sum_{q \in F} z_{j}^{q} a_{i j} \quad \forall i \in I, \forall j \in S \\
\sum_{i \in I} d_{i} x_{i j}+\sum_{l \in S, q \in F}\left(f_{l j}^{q}-f_{j l}^{q}\right)-f_{j N}=0 \quad \forall j \in S \\
y_{j l}^{q} \sum_{(k, h) \in C_{j l}} \frac{f_{k h}^{q}}{u_{k h}} \leq 1 \quad \forall j, l \in S, \forall q \in F .
\end{gathered}
$$

To limit to B the maximum number of channels assigned to a mesh node a new set of constraints must be added:

$$
\sum_{q \in F} z_{j}^{q} \leq B \quad \forall j \in S,
$$

and to define new variables $t_{j}$ we add:

$$
t_{j} \leq z_{j}^{q} \quad \forall j \in S, \forall q \in F .
$$

The new model will be referred to as Multi-Channel Model (MCM).

\section{$3 \quad$ Numerical Results}

In this section we test the sensitivity of the models proposed in previous section to different parameters like the number of candidate sites, the traffic demands from the MCs and the installation costs. To this end, we have implemented a generator of WMN topologies which considers specific parameter settings and makes some assumptions on propagation and device features. Obviously, these assumptions do not affect the proposed model which is general and can be applied to any problem instance. The generator considers a square area with edge $L=1000 \mathrm{~m}$, 
and it randomly draws the position of $m$ CSs and of $n=100$ TPs. The coverage area of a mesh node is assumed to be a circular coverage region with radius $R_{A}=100 \mathrm{~m}$. Only feasible instances where each TP is covered by at least one CS are considered. The wireless range of wireless backbone links is $R_{B}=250$ $\mathrm{m}$, while the capacities of the access links, $v_{j}$ and backbone links, $u_{j l}$, are both set to $54 \mathrm{Mb} / \mathrm{s}$ for all $j$ and $l$. The capacity of links connecting MAPs to the wired network is $M=128 \mathrm{Mb} / \mathrm{s}$, while the ratio between the cost of a $\mathrm{MR}$ and a MAP is $\beta$ ( $\beta=1 / 10$ unless otherwise specified).

All the results commented hereafter are the optimal solutions of the considered instances obtained formalizing the model in AMPL [16] and solving it with CPLEX [15] using workstations equipped with a AMD Athlon (TM) processor with CPUs operating at $1.2 \mathrm{GHz}$, and with $1024 \mathrm{Mbyte}$ of RAM.

\subsection{Fixed Rate Model}

Once assigned the number and the positions of either CS and TP, the quality of the deployed WMN and consequently the overall installation cost depends on two parameters: the traffic demand $d$ of the MCs and the ratio between the MR and MAP installation costs $\beta$. In this section we analyze the sensitivity of the proposed model to these parameters.

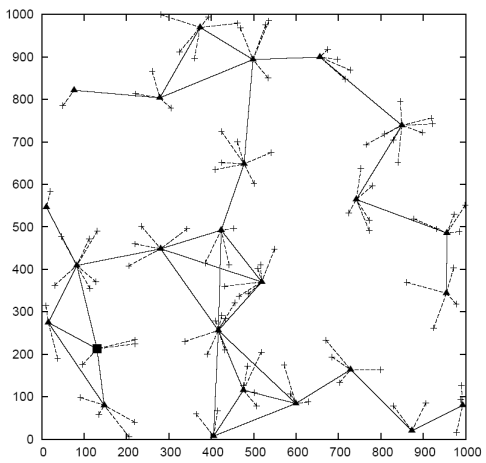

(a) $\mathrm{d}=600 \mathrm{~Kb} / \mathrm{s}$

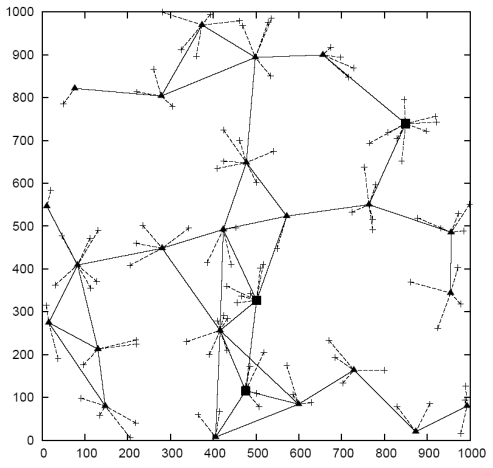

(b) $\mathrm{d}=3 \mathrm{Mb} / \mathrm{s}$

Fig. 2. Sample WMNs planned by the FRM with increasing traffic demands of the MCs and finite capacity of the installed MAPs $(M=128 \mathrm{Mb} / \mathrm{s})$. Standard setting of the topological parameters.

Effect of the Traffic Demands. Figure 2 reports an example of the planned networks when applying the FRM to the same instance with two different requirements on the end user traffic, $d=600 \mathrm{~Kb} / \mathrm{s}$ and $d=3 \mathrm{Mb} / \mathrm{s}$ for all MCs.

Table 1 analyze the characteristics of the solutions of the FRM when varying the number of candidate sites. The results presented are obtained averaging each point on 10 instances of network topology. For each couple $(m, d)$ the tables 
Table 1. Solutions provided by the FRM

\begin{tabular}{c|c|c|c|c|c|c|c|c|} 
& \multicolumn{4}{|c|}{$d=600 K b / \mathbf{s}$} & \multicolumn{4}{c|}{$d=3 M b / s$} \\
\cline { 2 - 9 } & MAP & MR & Links & Time (s) & MAP & MR & Links & Time (s) \\
\hline $\mathbf{m = 3 0}$ & 2.25 & 23.75 & 21.50 & 0.4 & 4 & 23.65 & 20.20 & 0.63 \\
\hline $\mathbf{m = 4 0}$ & 1.45 & 24 & 22.55 & 1.43 & 3.40 & 23.75 & 21.00 & 10.93 \\
\hline $\mathbf{m = 5 0}$ & 1.25 & 24.15 & 22.9 & 4.69 & 3.25 & 23.95 & 21.55 & 32.88 \\
\hline
\end{tabular}

report the number of installed MRs, the number of installed MAPs, the number of wireless links of the WDS and the processing time to get the optimal solution.

Two main results come from the observation of the table: first, the very same effect of traffic increase observed in Figure2 2 is evident also on averaged results, in fact the number of installed MAPs increases when increasing the traffic demands. Second, for a given traffic value, increasing the number of CS to 50 augments the probability for a MC to be connected to a MAP through a multi hop wireless path, therefore the model tends to install less MAPs and more MRs. On the other side, if the number of CS is lower (30), the model installs more MAPs since not all the MCs can be connected to the installed MAPs through multi hop wireless paths. In other words, with high $m$ the solution space is bigger and the model favors those solutions providing connectivity which have a lower impact on the network cost, i.e., those installing more MRs than MAPs.

Effect of the Cost Parameter. The number of installed MAPs and MRs intuitively depends on the installation cost ratio between a simple wireless router and a mesh access point.

Table 2. Solutions provided by the FRM when varying the installation cost ratio $\beta$. Number of CS $m=30$.

\begin{tabular}{c|c|c|c|||c|c|c|}
\multirow{2}{*}{$\beta$} & \multicolumn{2}{|c||}{$\mathbf{d}=\mathbf{6 0 0 K b} / \mathbf{s}$} & \multicolumn{3}{c|}{$d=3 \mathrm{Mb} / \mathbf{s}$} \\
\cline { 2 - 7 }$\beta$ & MAP & MR & Links & MAP & MR & Links \\
\hline $\mathbf{1 / 1 0}$ & 2.10 & 23.40 & 21.30 & 3.80 & 23.30 & 20.20 \\
\hline $\mathbf{1 / 7}$ & 2.40 & 24.00 & 21.60 & 4.10 & 23.80 & 20.10 \\
\hline $\mathbf{1 / 5}$ & 2.40 & 24.30 & 22.70 & 4.10 & 23.80 & 20.10 \\
\hline $\mathbf{1 / 3}$ & 2.40 & 24.30 & 21.60 & 4.10 & 23.80 & 20.10 \\
\hline $\mathbf{1 / 2}$ & 2.80 & 23.60 & 20.80 & 4.20 & 23.60 & 19.60 \\
\hline
\end{tabular}

Table2 2 reports the characteristics of the solutions when varying the parameter $\beta$ for different values of the offered traffic $d$. The results reported in the table shows that if the cost for installing a MAP decreases with respect to the cost of MRs, the proposed model tends to install more MAPs. However, the differences observed with different cost ratios are much smaller than what one could expect. 
Table 3. Quality of the solutions provided by the RAM

\begin{tabular}{c|c|c|c|c|c|c|c|c|}
\multirow{2}{*}{} & \multicolumn{4}{|c|}{$\mathbf{d = 2 0 0 K b / s}$} & \multicolumn{4}{c|}{$\mathbf{d = 6 0 0 K b / s ~}$} \\
\cline { 2 - 9 } & MAP & MR & Links & Time (s) & MAP & MR & Links & Time (s) \\
\hline $\mathbf{m = 3 0}$ & 2.80 & 23.80 & 21.00 & 2.24 & 2.80 & 25.40 & 22.60 & 2.24 \\
\hline $\mathbf{m = 4 0}$ & 1.70 & 24.60 & 22.90 & 9.66 & 1.70 & 26.00 & 24.30 & 13.25 \\
\hline $\mathbf{m = 5 0}$ & 1.20 & 24.40 & 23.20 & 46.83 & 1.20 & 26.10 & 24.90 & 61.37 \\
\hline
\end{tabular}

The reason is that in the considered scenario the optimization process is driven mainly by the capacity constraints. We obtained results (not shown here) with a bigger difference in the number of installed MAPs by letting higher capacity for the links connecting MAPs (setting high values to parameter $M$ ).

\subsection{Rate Adaptation Model}

In real wireless networks, the capacity of a given wireless link depends on the distance between transmitter and receiver. The RAM endorses this fact by defining three capacity regions around a MR (and MAP) and assigning the link between $\mathrm{MC}$ and MR (or MAP) an increasing capacity when getting nearer to the MR (or MAP) location. The rate values $v_{i}^{k}=v^{k} \forall j$ adopted to obtain numerical results emulates IEEE $802.11 \mathrm{~g}$ transmission and depends on the distance $r$ : $0 m \leq r \leq 30 m v^{k}=36 \mathrm{Mb} / \mathrm{s}, 30 m<r \leq 60 m v^{k}=18 \mathrm{Mb} / \mathrm{s}, 60 \mathrm{~m}<r \leq 100 \mathrm{~m}$ $v^{k}=2 \mathrm{Mb} / \mathrm{s}$.

The behavior of the RAM is similar to the one of the FRM in terms of sensitivity to the model parameters. Table 3 summarizes the characteristics of the solutions of the RAM when varying the number of candidate sites. The traffic offered by the MCs is lower with respect to the one used to test the FRM since the link rates are in the average lower then those in the FTR case. As a result, we observed that higher values of $d$ may lead to unfeasible instances. The results obtained highlight a behavior of the RAM very similar to the one already observed for the FRM in the same configuration.

\subsection{Interference Aware Model}

The IAM considers the impact of the interference on the access capacity, through constraints (14), and the capacities wireless links, through constraints (13). Table 4 reports the results obtained with the IAM for the special case in which rate adaptation is not included. The parameter settings are the same adopted for the FRM and therefore results in Table 4 can be directly compared with those reported in Table 1 Sets $C_{j l}$ have been obtained considering the IEEE 802.11 multiple access scheme.

We observe that the number of MAPs installed is remarkably higher and the number of links lower with respect to the FRM case. This is due to the capacity reduction of wireless links that favors solutions where MRs and MAPs are interconnected through paths with a small number of hops. In fact, with short paths between MRs and MAPs the effect of interference is weaker. Obviously, short paths 
Table 4. Solutions provided by the IAM without rate adaptation

\begin{tabular}{c|c|c|c|c|c|c|c|c|} 
& \multicolumn{4}{|c|}{$\mathbf{d = 6 0 0 K b / s}$} & \multicolumn{4}{c|}{ d=3Mb/s } \\
\cline { 2 - 9 } & MAP & MR & Links & Time (s) & MAP & MR & Links & Time (s) \\
\hline $\mathbf{m = 3 0}$ & 3.40 & 22.20 & 19.40 & 4.37 & 8.50 & 22.00 & 14.20 & 4.16 \\
\hline $\mathbf{m = 4 0}$ & 2.50 & 23.10 & 20.90 & 258.09 & 7.80 & 22.50 & 16.10 & 53.98 \\
\hline $\mathbf{m = 5 0}$ & 2.30 & 23.40 & 21.60 & $1,706.63$ & 7.70 & 23.40 & 17.80 & $1,345.32$ \\
\hline
\end{tabular}

Table 5. Solutions provided by the MCM without rate adaptation

\begin{tabular}{c|c|c|c|c|c|c|c|c|}
\multirow{2}{*}{} & \multicolumn{4}{|c|}{$\mathbf{d = 6 0 0 K b / s}$} & \multicolumn{4}{c|}{$\mathbf{d = 3 M b} / \mathbf{s}$} \\
\cline { 2 - 9 } & MAP & MR & Links & Time (s) & MAP & MR & Links & Time (s) \\
\hline $\mathbf{m = 3 0}$ & 2.25 & 23.75 & 21.60 & 2.99 & 4.00 & 23.65 & 20.55 & 9.79 \\
\hline $\mathbf{m = 4 0}$ & 1.45 & 24.00 & 22.60 & 73.43 & 3.40 & 23.75 & 21.30 & 141.18 \\
\hline $\mathbf{m = 5 0}$ & 1.25 & 24.05 & 22.85 & 393.38 & 3.30 & 23.65 & 21.35 & 673.52 \\
\hline
\end{tabular}

require a higher number of MAPs. Another relevant difference between the FRM and the IAM results is the computation time which is much higher for IAM in most of the cases. As expected this is due to the structure of constraints (13) which involve several flow variables simultaneously. A similar behavior can be observed considering the results (not shown here) obtained with IAM and rate adaptation.

\subsection{Multiple Channel Model}

The MCM adds to the planning problem the channel assignment to multi-radio devices. Table 5 reports the numerical results obtained by the MCM when considering $Q=11$ channels and $B=3$ radio interfaces, which are typical values for the IEEE 802.11a technology. We observe that the results are not very different from those obtained with the FRM (see Table 1) in terms of installed MAPs, MRs and links.

On the other side, the computation complexity of MCM is much higher than the one of the other models: considering a constraints of four hours on the computation time, we are able to obtain the optimum solution (reported in the table) only in the $80 \%$ of the instances. Therefore, the MCM should be adopted when planning multi-channel/multi-radio WMNs only in case the number of available channels and interfaces is very limited. In the other cases the FRM/RAM models can be safely used for planning the network and frequency assignment can be optimized in a second phase [4].

\section{Conclusion}

In this paper we proposed an optimization model based on mathematical programming whose objective function is the minimization of the overall network 
installation cost while taking into account the coverage of the end users, the wireless connectivity in the wireless distribution system and the management of the traffic flows. Technology dependent issues such as rate adaptation and interference effect have been considered.

To test the quality of the solutions provided by the model, we generated synthetic instances of WMNs and solved them to the optimum using AMPL/CPLEX varying several network parameters. The numerical results we gathered show that the model is able to capture the effect on the network configuration of all these parameters, providing a promising framework for the planning of WMNs.

\section{References}

1. I.F. Akyildiz, X. Wang, I. Kiyon, A Survey on Wireless Mesh Networks, IEEE Com. Mag., Sept. 2005, Vol. 43, No. 9, pp. 23-30.

2. J. So, N. Vaidya, Multi-Channel MAC for ad hoc Networks: Handling MultiChannel Hidden Terminals using a Single Transceiver, ACM MOBIHOC 2004, pp. 222-233.

3. A. Adya et al., A Multi-Radio Unification Protocol for IEEE 802.11 Wireless Networks, IEEE BROADNETS 2004, pp. 344-354.

4. A.K. Das, H.M.K. Alazemi, R. Vijayakumar, S. Roy, Optimization models for fixed channel assignment in wireless mesh networks with multiple radios, IEEE SECON 2005, pp. 463-474.

5. M. Alicherry, R. Bhatia, L. E. Li, Multi-radio, multi-channel communication: Joint channel assignment and routing for throughput optimization in multi-radio wireless mesh networks, ACM MOBICOM 2005, pp. 58-72.

6. A. Raniwala, K. Gopalan, and T. C. Chiueh, Centralized channel assignment and routing algorithms for multichannel wireless mesh networks, ACM MC2R, Apr. 2004, Vol. 8, No. 2, pp. 50-65.

7. E. Amaldi, A. Capone, F. Malucelli, Planning UMTS Base Station Location: Optimization Models with Power Control and Algorithms, IEEE Trans. on Wireless Comm., Sept. 2003, Vol. 2, No. 5, pp. 939-952.

8. S. Bosio, A. Capone, M. Cesana, Radio Planning of Wireless Local Area Networks, ACM/IEEE Trans. on Net., to appear.

9. E. Amaldi, A. Capone, F. Malucelli, C. Mannino, Optimization Problems and Models for Planning Cellular Networks, in Handbook of Optimization in Telecommunications, Kluver Academic Publishers, 2006.

10. M. Pioro, D. Medhi, Routing, Flow, and Capacity Design in Communication and Computer Networks, Morgan Kaufmann Publishers, 2004.

11. R. Chandra, L. Qiu, K. Jain, M. Mahdian, Optimizing the placement of integration points in multi-hop wireless networks, IEEE ICNP 2004, pp. 271-282.

12. M. Kodialam, T. Nandagopal, Characterizing the Capacity Region in Multi-Radio Multi-Channel Wireless Mesh Networks, ACM Mobicom 2005, pp. 73-87.

13. Y. Bejerano, Efficient integration of multi-hop wireless and wired networks with QoS constraints, ACM MOBICOM 2002, pp. 215-226.

14. P. Gupta and P.R Kumar, The capacity of wireless networks, IEEE Trans. on Inf. Theory, Mar. 2000, Vol. 46, No. 2, pp. 388-404.

15. ILOG CPLEX 8.0 user's manual, 2002.

16. R. Fourer, D. M. Gay, and B. W. Kernighan, AMPL, A modeling language for mathematical programming, 1993. 